\title{
Fisiología de la tiroides e hipotiroidismo en el embarazo. Revisión de tema
}

\section{Thyroid physiology and hypothyroidism in pregnancy. A review}

\author{
Myriam Vanessa Rueda-Galvis ${ }^{1}$ (iD, Carlos Alfonso Builes-Barrera² ${ }^{\text {iD }}$
}

\begin{abstract}
Resumen. Durante el embarazo se generan múltiples cambios fisiológicos a nivel hormonal para llevar a cabo de manera satisfactoria la gestación. Uno de los ejes hormonales con cambios más importantes que repercuten de manera directa en el desarrollo fetal y bienestar materno es el tiroideo, el cual presenta modificaciones para lograr suplir las necesidades de hormona tiroidea tanto materna como fetal, principalmente en las primeras etapas del embarazo. Entre estas, se describen cambios en la cantidad de proteínas transportadoras de hormonas, aumento en el estímulo y producción de hormonas tiroideas, incremento del aclaramiento renal de yodo y alteración en la actividad de las desyodinasas. Estos mecanismos ofrecen suficiente hormona tiroidea al feto, el cual es dependiente del aporte materno. Un desajuste en cualquiera de estos mecanismos, puede conducir al desarrollo de hipotiroidismo con múltiples complicaciones, como la pérdida del embarazo e hipertensión gestacional, entre otras. Una tamización oportuna y un tratamiento temprano pueden evitar estos desenlaces adversos. De ahí la necesidad fundamental de conocer y comprender el comportamiento del eje tiroideo en la gestación.
\end{abstract}

Palabras clave: glándula tiroides, embarazo, gestación, hipotiroidismo, fisiología.

Abstract. During pregnancy, multiple physiological changes are generated at the hormonal level to successfully carry out pregnancy. One of the hormonal axes with the most important changes that have a direct impact on fetal development and maternal well-being is the thyroid axis, which presents multiple modifications to reach the needs of thyroid hormone for both the mother and the fetus, primarily in the early stages of pregnancy. Changes in the amount of hormone transport proteins, increased stimulation and production of thyroid hormones, increased re-

\footnotetext{
${ }^{1}$ Médica, Especialista en Medicina Interna. Residente de Endocrinología, Departamento de Medicina Interna, Sección de Endocrinología y Metabolismo, Facultad de Medicina, Universidad de Antioquia. Medellín, Colombia. E-mail: vanessa.rueda@ udea.edu.co.

2 Médico, Especialista en Medicina Interna, Especialista en Endocrinología. Departamento de Medicina Interna, Sección de Endocrinología y Metabolismo, Facultad de Medicina, Universidad de Antioquia, Hospital San Vicente Fundación. Medellín, Colombia.

Conflicto de interés: los autores declaran que no tienen conflicto de interés.

Medicina \& Laboratorio 2022;26:15-33. https://doi.org/10.36384/01232576.557.

Recibido el 2 de noviembre de 2021; aceptado el 2 de diciembre de 2021. Editora Médica Colombiana S.A., 2022 ${ }^{\circ}$.
} 
nal clearance of iodine, and alteration in deiodinase activity are included within these modifications. These mechanisms offer enough thyroid hormone to the fetus, which is dependent on the maternal supply. An imbalance in any of these can lead to the development of hypothyroidism with multiple complications, such as pregnancy loss and gestational hypertension, among others. Timely screening and early treatment can avoid these adverse outcomes, hence the importance of knowing and understanding the regulation of the thyroid axis in pregnancy.

Keywords: thyroid gland, pregnancy, gestation, hypothyroidism, physiology.

\section{Introducción}

La hormona tiroidea es fundamental para el desarrollo normal del infante y el mantenimiento de la salud del adulto. Múltiples cambios ocurren a nivel hormonal en la mujer embarazada, que pueden impactar en el desarrollo de un embarazo sano [1]. La gestación genera grandes cambios en la tiroides; se estima que existe un crecimiento promedio del $10 \%$ al $40 \%$ de la glándula tiroides, con un incremento asociado de la producción de hormonas tiroideas hasta del $50 \%$, con mayor necesidad de yodo para su producción $[2,3]$.

En las últimas décadas se ha descrito que la disfunción tiroidea es el trastorno endocrino más prevalente durante el embarazo, con $0,2 \%$ para el caso de la enfermedad de Graves y hasta 3\% para el hipotiroidismo [4]. Tanto la función tiroidea de la madre como la del feto juegan un papel fundamental; la de la madre en el desarrollo temprano de la gestación [1], ya que el crecimiento y desarrollo del feto es dependiente de esta hasta mediados del embarazo (semana 16 a 20), con un aporte progresivo fetal en las fases tardías, hasta que el feto inicia su propia producción hormonal. De ahí la importancia de una adecuada homeostasis hormonal de la gestante [5].

\section{Fisiología de la tiroides en el embarazo}

Para comprender los cambios en las hormonas tiroideas durante el embarazo, se debe entender la fisiología del embarazo y los ajustes hormonales tiroideos que la acompañan; principalmente el aumento de la globulina fijadora de tiroxina (TBG, del inglés, Thyroxine-Binding Globulin) y la estimulación del receptor de tirotropina (TSHR) mediado por la gonadotropina coriónica humana [1] (tabla 1). Se genera una demanda de hormona tiroidea, lo que lleva a un incremento hasta de un $50 \%$ en la producción de tiroxina, con el fin de mantener el estado eutiroideo, lo cual solo puede ser logrado en condiciones de suficiencia de yodo y ausencia de un proceso autoinmune tiroideo [6].

\section{Globulina fijadora de tiroxina}

La TGB es una glicoproteína de 54 kDa sintetizada a nivel hepático, como una cadena polipeptídica de 415 aminoácidos, con un sitio de unión único a la yodotironina, y una especificidad mayor para la tiroxina (T4) que para la triyodotironina (T3) [1]. Las hormonas tiroideas son transportadas en el torrente sanguíneo por la TGB, transtirretina y albúmina [7]. Por otra parte, la produc- 


\begin{tabular}{ll}
\hline Tabla 1. Cambios fisiológicos en el embarazo & \\
\hline Sustancia & Efecto \\
\hline Globulina fijadora de tiroxina & Aumenta \\
Albúmina & Disminuye \\
hCG & Aumenta y estimula la glándula tiroides \\
Hormonas tiroideas totales & Aumentan \\
& D1: disminuye su actividad \\
Desyodinasas & D2: se mantiene \\
\hline Yodo renal & D3: aumenta su expresión placentaria \\
Tiroglobulina & Aclaramiento incrementado \\
\hline
\end{tabular}

hCG: gonadotropina coriónica humana; D: desyodinasa.

ción de TGB puede ser modificada por niveles de estrógeno, corticosteroides o insuficiencia hepática.

El embarazo es un estado de hiperestrogenemia, donde el exceso de estrógeno provoca un aumento de la producción de TGB, además, favorece la sialilación de la misma, lo que disminuye la depuración global de la proteína transportadora, generando una duplicación de los niveles de TGB disponibles. El exceso de TGB ocasiona una mayor unión de tiroxina, disminuyendo así la cantidad de hormona tiroidea libre disponible, aumentando la cantidad de hormonas tiroideas totales, lo cual desencadena una mayor producción de la hormona estimulante de la tiroides (TSH) [6]. Para mantener las concentraciones adecuadas de hormona libre, se debe aumentar la producción de T4 y T3 (figura 1).

Los niveles de hormonas tiroideas totales aumentan aproximadamente un $30 \%$ a $50 \%$ antes de la semana $20 \mathrm{del}$ embarazo, momento en el que se estabilizan, en parte, gracias a la maduración progresiva del eje hipotálamo-hipófisis-tiroideo fetal [9]. Esto se manifiesta como una concentración de T4 total incrementada en el primer trimestre (debido al aumento de TGB), con posterior disminución en el segundo y tercer trimestre, lo cual impacta de manera consecutiva, aumentando los niveles de la TSH durante la fase tardía del embarazo $[6,10]$.

\section{Gonadotropina coriónica humana}

La gonadotropina coriónica humana (hCG) es elaborada en el sincitiotrofoblasto, ovarios y testículos. Su función al inicio del embarazo es un efecto trófico sobre el cuerpo lúteo, para mantener la producción de progesterona, hasta que la placenta sea capaz de sintetizarla [11].

La hCG es una glicoproteína de la misma familia de la TSH, con una subunidad alfa común para la TSH, la hormona folículo estimulante (FSH) y la hormona luteinizante (LH), pero con una subunidad beta única. De modo que la hCG es capaz de estimular de manera débil la producción de hormona tiroidea, al unirse a su receptor en la glándula tiroides [12].

Los niveles de hCG aumentan progresivamente después de la fertilización, 


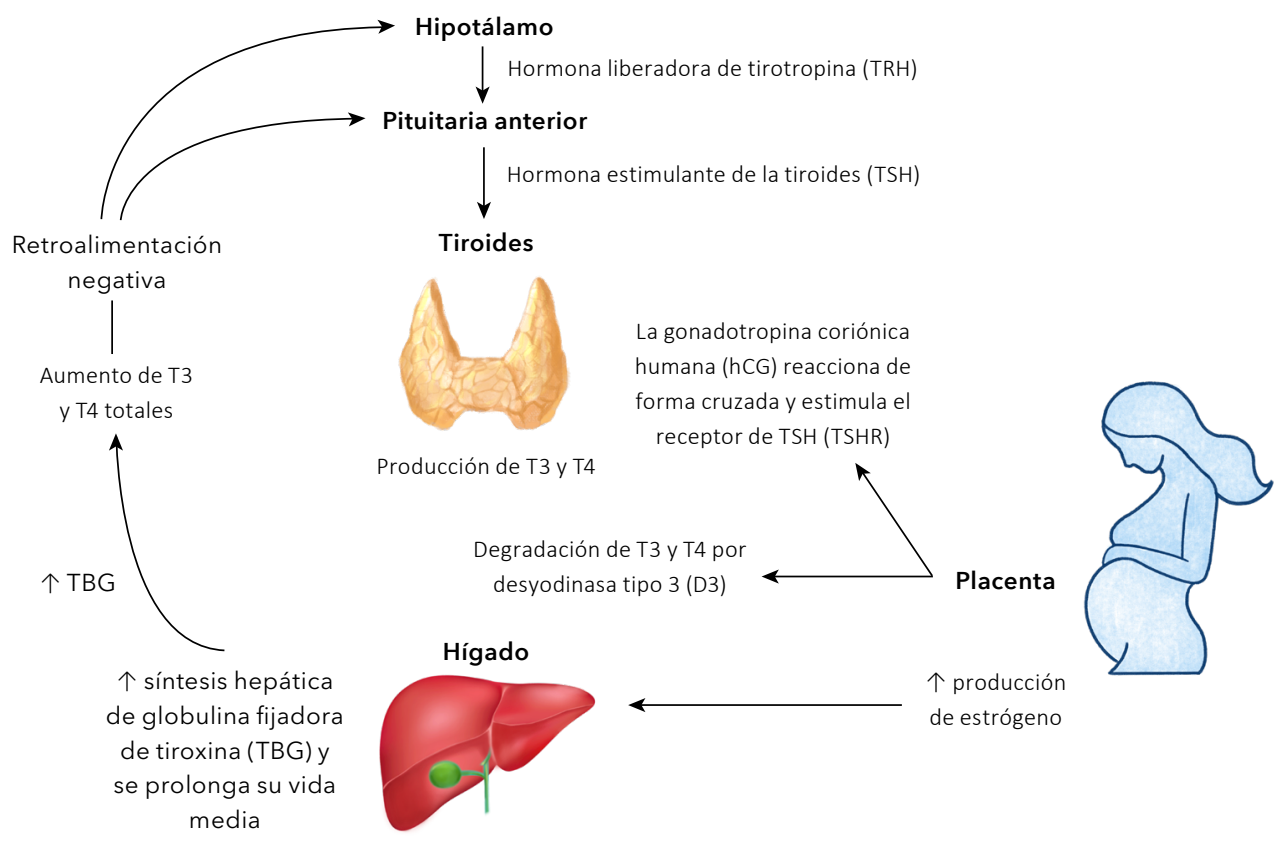

Figura 1. Fisiología tiroidea en el embarazo. Tomado y adaptado [8].

con un pico hacia la semana 12 de gestación, generando incrementos en los niveles de T4 y T3 totales, y en sus niveles libres en menor proporción, así como una disminución consecuente de los niveles de TSH [12]. Incluso en los casos de exceso de hCG (hiperémesis gravídica, embarazo múltiple), la TSH puede llegar a suprimirse, con niveles de tiroxina libre (T4L) elevados, generando un hipertiroidismo que habitualmente es transitorio hasta en el $20 \%$ de los embarazos [13]. Después de la semana 12, los niveles de hCG tienden a disminuir, alcanzando una meseta durante el resto de la gestación.

Los niveles de TSH son entonces regulados en el embarazo por la disponibilidad de hormonas tiroideas circulantes, pero también por los niveles de hCG. Por su parte, los valores de T4L reflejan la carga de hormona disponible en la madre y el feto, la cual, a diferencia de la TSH no está influenciada por la hCG, sino por la saturación de yodo y la duración del embarazo.

\section{Yodotironina desyodinasas}

La disponibilidad de hormonas tiroideas en el feto no depende solo de la transferencia placentaria y la producción por la glándula tiroidea fetal, sino también del metabolismo periférico. Es por eso que las desyodinasas son esenciales en la homeostasis hormonal fetal [6]. Estas son enzimas óxido reductasas humanas (D1, D2 y D3), que también se conocen como selenio enzimas. La D1 por su parte, cataliza la conversión periférica de T4 a T3 e inactiva los conjugados sulfatados de T4. La D2 (enzima activadora) ubicada en el retículo endoplásmico, cataliza la conversión de 
T4 a T3 a nivel citoplasmático, y la D3 (enzima desactivadora) previene la activación de T4 al convertirla en T3r (T3 reversa) [14].

Durante la gestación, la expresión de desyodinasas cambia. La actividad de D1 disminuye, y como consecuencia los niveles de T3 en el feto son bajos, mientras que los niveles de T3r y T3 sulfatada están incrementados. No se conoce hasta la fecha la utilidad biológica de mantener niveles de T3 fetales bajos [15]. Por otra parte, la D3, que es la que predomina al inicio de la gestación, y la D2 durante el primer trimestre [7], se encuentran en el cerebro fetal desde etapas tempranas (semana 7), y cumplen un rol importante al definir la cantidad de T3 disponible en el cerebro y la pituitaria fetal.

Durante el embarazo, las proteínas transportadoras como la transtirretina y la albúmina, en conjunto con la $\alpha 1$ antitripsina y la $\beta 1$-glicoproteína ácida, se secretan de manera continua al torrente materno-fetal [16]. Estas proteínas se encargan de regular el microambiente placentario para favorecer el paso de hormonas tiroideas a la circulación fetal. La transtirretina por su parte, disminuye la desyodación de las hormonas tiroideas, lo que permite un paso elevado de hormonas tiroideas que pueden llegar a través de la placenta, líquido amniótico y cordón umbilical a la circulación fetal $[7,16]$; por lo tanto, para proteger al feto de la exposición hormonal inapropiada de hormonas tiroideas, es esencial la expresión ubicua de desyodinasas en el feto [17]. Esto se logra con la expresión clave de D3 placentaria, que protege al feto contra el exceso de exposición de T4 de origen materno.

A pesar de que los niveles de T3 circulante pueden ser bajos, en el cerebro fetal de 26 semanas son del $80 \%$. De modo que la relación entre las desyodinasas del feto y la placenta tratan de mantener niveles circulantes de T3 bajos, sin embargo, el cerebro fetal desarrolla mecanismos para mantener T3 óptimos para el desarrollo neurológico normal [18].

\section{Aclaramiento renal de yodo}

El embarazo incrementa la filtración glomerular ocasionando que se aumente el aclaramiento renal de yodo. Esto favorece una menor disponibilidad de yodo circulante para la formación de hormonas tiroideas, lo que lleva a un mayor riesgo de hipotiroidismo y bocio durante el embarazo [19].

\section{Transporte de hormonas tiroideas}

Durante el embarazo, las hormonas tiroideas, al igual que en cualquier otro tejido diana, requieren transportadores de membrana para el desplazamiento hormonal hasta el núcleo. La placenta expresa múltiples transportadores de membrana como LAT1, LAT2, MCT8, MCT10, OATP1A2 y OATP4A1, que favorecen el intercambio materno placentario de hormonas tiroideas, principalmente durante las primeras semanas de la gestación [20].

\section{Tiroglobulinas}

La tiroglobulina (Tg) es una proteína de almacenamiento tiroideo y precursor de la síntesis de T3 y T4. Los estados de deficiencia de yodo y el embarazo, generan una correlación positiva entre la cantidad de $\mathrm{Tg}$ y el volumen de la glándula tiroidea, ocasionando bocio del embarazo en el $15 \%$ de los casos $[6,21]$. 
Esta proteína tiende a incrementar hacia la mitad del embarazo, y aún más cerca de las 36 semanas de gestación, retornando a sus valores normales en el posparto [22]. Se ha intentado usar las mediciones de Tg como un marcador materno de deficiencia de yodo, sin embargo, los resultados de su determinación tienen alta variabilidad entre los ensayos, y están influenciados por la edad y el método de medición, limitando su utilidad [6].

\section{Fisiología de la tiroides del feto}

Las hormonas tiroideas en el feto son fundamentales para el desarrollo temprano del cerebro, crecimiento somático y maduración ósea. Adicionalmente, son importantes para la supervivencia y diferenciación celular, la homeostasis celular y la regulación metabólica dentro de múltiples tipos de tejidos y órganos [23]. Existen dos fuentes importantes de hormonas tiroideas en la gestación; la primera, la glándula tiroidea materna, y la segunda, la glándula fetal en maduración, las cuales dependen de la ingesta adecuada y suficiente de yodo [6]. La T4 materna es especialmente importante en la primera mitad del embarazo, cuando el feto aún no produce hormona, y su disponibilidad va a depender del paso a través de la placenta como se describió con anterioridad [19].

La maduración de la función tiroidea fetal incluye un complejo proceso que implica la organogénesis, maduración del eje hipotálamo-hipófisis-tiroides, así como el metabolismo hormonal. La glándula tiroides se desarrolla a partir del piso de la faringe primitiva y la cuarta bolsa faríngea. Este proceso es completado entre la semana 12 a 14 de gestación. En esta etapa, se pueden ver pequeños precursores de folículos y Tg en los espacios foliculares. Entre las semanas 10 a 12 de la gestación, se expresa por primera vez la TSH fetal, y la glándula tiroidea fetal inicia la concentración de yodo y la síntesis de yodotironinas. Sin embargo, es hasta la semana 20 de gestación cuando se sintetiza la hormona tiroidea, incrementándose progresivamente a partir de ese momento [24].

Al finalizar la gestación, el feto tiene una producción independiente de hormona tiroidea y su concentración varía sustancialmente de la materna, con niveles de TSH más altos, T4L más bajos, y T3 alrededor de la mitad respecto a la materna [6]. En el nacimiento inmediato se aumentan exageradamente los niveles de TSH hasta valores de $50 \mathrm{mUI} / \mathrm{L}$, los cuales posteriormente descienden hasta alrededor de $10 \mathrm{mUI} / \mathrm{L}$ al segundo día del nacimiento, con aumentos de T3 y T4 por encima de los valores fisiológicos del adulto [24].

\section{Consumo de yodo y embarazo}

El aumento del aclaramiento renal del yodo y la mayor producción de T4 de origen materno (hasta en un $50 \%$ ) durante el embarazo, lleva a la necesidad de aumentar el consumo de yodo proveniente de la dieta [6].

Dentro de las recomendaciones mundiales, la Organización Mundial de la Salud (OMS) sugiere el consumo mínimo de $250 \mu \mathrm{g}$ de yodo al día durante el embarazo y la lactancia. La cantidad máxima tolerable de ingesta de yodo en mujeres embarazadas es de $600 \mu \mathrm{g}$ a $1.100 \mu \mathrm{g}$ al día $[25,26]$. Además, recomienda mantener niveles urinarios de yodo durante el embarazo con valores alrededor de $149 \mu \mathrm{g} / \mathrm{L}$ a $249 \mu \mathrm{g} / \mathrm{L}$, asociados a niveles de consumo ópti$\operatorname{mos}[27]$. 
El monitoreo regular de yodo es importante para mantener la suficiencia de yodo, primordialmente en el embarazo, por lo que en países europeos se recomienda esta práctica donde existen grupos de alto riesgo, debido a la falta de políticas de yodación, control inadecuado de estas políticas, o a la disminución en el uso de la sal en la dieta [28]. Las estadísticas nacionales reportadas en Colombia en las encuestas nacionales de nutrición, muestran suficiencia de yodo con una mediana de $354 \mu \mathrm{g} / \mathrm{L}$ para los años 2013 a 2014 [29], por lo que es poco probable que en esta región se presente una deficiencia de yodo durante el embarazo, y por lo tanto, no existe una recomendación de suplementación de yodo adicional durante la gestación.

\section{Anticuerpos antitiroideos, autoinmunidad y embarazo}

El estado de embarazo es una situación fisiológica que obliga a la generación de inmunotolerancia en la madre para lograr una inmunosupresión parcial, que no rechace los antígenos fetales y permita el mantenimiento de la gestación [30]. La respuesta inmune materna en forma de inmunosupresión evita el rechazo del embarazo. En este estado se presenta incremento de las células $T$ reguladoras y citoquinas circulantes [31], habitualmente asociado a un desequilibrio entre la inmunidad Th1 y Th2, las células Th17 y las células $T$ reguladoras [32]. La complejidad de la relación madre-feto está aún en exploración, sin embargo, un desequilibrio es quizá la causa más frecuente de abortos espontáneos [6].

En el embarazo ocurren cambios que conducen a la tolerancia inmunológica fetal, el cambio primordial es la expresión diferente del HLA (antíge- nos leucocitarios humanos), las células trofoblásticas no expresan el antígeno mayor de histocompatibilidad (MHC), MHC I y II, pero expresan el Ib (HLAE, HLA-G), que se encarga de generar una inhibición de las natural killers y linfocitos T CD8+. Además, se genera la inhibición de la activación del complemento y apoptosis de células T activadas, mediada por el ligando Fas y la expresión del ligando inductor de apoptosis relacionado con TNF- $\alpha$, así como un cambio hacia la respuesta inmune Th2 (linfocito T helper 2), debido al aumento de la secreción de estrógenos y progesterona [6,33].

Los estados de autoinmunidad tiroidea se desarrollan usualmente por falla a la tolerancia de las células B y $\mathrm{T}$ a los autoantígenos [33], lo cual genera una respuesta humoral y celular que termina afectando el tejido glandular. La autoinmunidad de la tiroides ha sido relacionada con 4 grupos de genes: complejo mayor de histocompatibilidad, sobre todo polimorfismos en HLA I y HLA II; también los genes encargados de la respuesta de activación (CTL-4) e inactivación (PTPN22) de las células $T$; el grupo que influencia otras partes de la respuesta inmune (por ejemplo, CD40 y CD26), que activan las células $B$ presentadoras de antígenos y las natural killers; $y$, finalmente, los autoantígenos tiroideos que determinan la especificidad tisular, como los genes que codifican el receptor de TSH y la tirosinasa [33].

En estado de gestación es común la expresión de autoinmunidad tiroidea, se han reportado prevalencias entre $5,1 \%$ al $12,4 \%$ de anticuerpos antiperoxidasa tiroidea (anti-TPO) y antitiroglobulinas (anti-Tg) en países con suficiencia de yodo $[34,35]$. Los mecanismos propuestos por medio de los cuales los anticuerpos se relacionan con desenlaces 
adversos parecen ser tres: 1) niveles tiroideos bajos, que pasan desapercibidos en las primeras semanas del embarazo; 2) pérdida de la tolerancia inmunológica de la madre al feto; $y, 3$ ) aumento de la presencia de autoanticuerpos a mayor edad [6].

El principal riesgo está dado por el desarrollo de hipotiroidismo manifiesto en el embarazo y posparto, por lo tanto, se justifica una vigilancia activa de la TSH en estas gestantes $[6,36,37]$. Las mujeres eutiroideas con anti-TPO y/o anti-Tg tienen mayores incrementos de TSH a lo largo de la gestación, y hasta un 19\% pueden presentar niveles elevados al momento del parto [37].

Existe una relación demostrada entre la pérdida temprana del embarazo (antes de las 20 semanas) y la autoinmunidad en mujeres eutiroideas, con tasas de hasta el $36 \%$ en aquellas con expresión de anti-TPO, independiente de los niveles de TSH [38,39]. El metaanálisis realizado por Thangarantinam y colaboradores, demostró que la presencia de anti-TPO o anti-Tg se asocia con tasas más altas de aborto; otros autores también presentaron evidencia a favor de esta asociación [40-42].

La relación entre la presencia de anticuerpos tiroideos y el parto pretérmino (antes de la semana 37) tiene evidencia conflictiva. En algunos estudios no se encontró relación alguna $[43,44]$, no obstante, otros demostraron evidencia a favor $[22,42,45]$, con un riesgo relativo de 1,41 (IC95\% 7,93$18,7)[43]$, muy similar a los hallazgos del estudio de Negro y colaboradores [44]. Lo mismo sucede con la relación entre autoinmunidad y el desarrollo cognitivo fetal, el tamaño cerebral, el trastorno de déficit de atención e hiperactividad, sin llegarse a resultados concluyentes [46-48].

\section{Niveles hormonales de tiroides en la gestación}

Al evaluar la función tiroidea materna en el embarazo, se deben medir los niveles de TSH y T4L (si se tiene el rango poblacional) o T4 total. El cambio más relevante introducido en las guías de American Thyroid Association (ATA), en la última actualización del 2017 respecto a la del 2011, fue la redefinición de los niveles normales de TSH [2]. En la anterior guía establecen un punto de corte de TSH de 2,5 mUI/L, con necesidad de inicio de reemplazo hormonal en presencia de valores inferiores. Sin embargo, a raíz de la revisión de cuatro grandes series con datos poblacionales en mujeres embarazadas, se ajustó el punto de corte [49-51]. La recomendación principal es obtener rangos propios para la población y tipo de estudio realizado. En ausencia de valores propios poblacionales, se deberían calcular en el primer trimestre reduciendo 0,5-1 $\mathrm{mUI} / \mathrm{L}$ del límite de referencia en el laboratorio, para población no gestante. Esto generaría un valor aproximado de 0,4 $\mathrm{mUI} / \mathrm{L}$ a $4 \mathrm{mUI} / \mathrm{L}$ en la mayoría de kits comerciales [2]. Durante el segundo y tercer trimestre, el valor de TSH tiende a aproximarse a los valores de mujeres no embarazadas.

Respecto a los niveles de tiroxina, retomando lo expuesto previamente en la fisiología de la tiroides en el embarazo, no es raro encontrar niveles de T4L bajos en relación a niveles de TSH normal, esto asociado a la inexactitud del ensayo (habitualmente inmunoensayos), la distribución de proteínas de unión TBG y la disminución de albúmina durante la gestación [52,53]. Los niveles de T4L parecen tener una tendencia a disminuir a lo largo de la gestación, pero sus niveles no son confiables a menos que sean realiza- 
dos mediante cromatografía líquida con espectrometría de masas, la cual genera una medición directa que está menos influenciada por las proteínas, pero es una tecnología más costosa y menos disponible [54].

\section{Definición y epidemiología del hipotiroidismo en la gestación}

El hipotiroidismo es una entidad común en las mujeres en edad gestacional, se estima una prevalencia del $2 \%$ en esta población [55]. Asimismo, es la disfunción más común en el embarazo asociada a la glándula tiroides. La ATA define el hipotiroidismo gestacional como niveles elevados de TSH con hipotiroxinemia asociada, para los rangos definidos para el trimestre en la población. Si no se cuenta con rangos propios poblacionales, el límite superior de TSH propuesto es de 4 $\mathrm{mUI} / \mathrm{L}$ con T4L baja, o TSH superior a $10 \mathrm{mUI} / \mathrm{L}$, independientemente de los niveles de hormonas tiroideas [2].

Para el hipotiroidismo gestacional manifiesto se estima una prevalencia del $0,3 \%$ al 0,5\% [6]. La prevalencia suele ser baja porque estas mujeres tienden a ser anovulatorias, con tasas más bajas de embarazo, además, el hipotiroidismo no tratado se complica con abortos espontáneos en el primer trimestre. La prevalencia de hipotiroidismo subclínico varía entre $4 \%$ a $17 \%$, y va a depender del punto de corte de TSH seleccionado en el primer trimestre, siendo mayor cuando se selecciona un punto de corte de TSH de 2,5 mUI/L [56]. Existe una entidad adicional en la que los niveles de TSH son normales, con niveles de T4L por debajo del percentil 2,5-5 para la población, y se conoce como hipotiroxinemia aislada [2].

\section{Manifestaciones del hipotiroidismo en el embarazo}

Las manifestaciones clínicas del hipotiroidismo durante el embarazo son similares a la de la población no gestante. Los síntomas pueden pasar inadvertidos o atribuirse a la gestación, si no se hace tamización activa. Las características clínicas están mediadas por un enlentecimiento de los procesos metabólicos a nivel celular, así como un incremento en el depósito de glicosaminoglicanos de matriz extracelular en el espacio intersticial [57], que se pueden manifestar como adinamia, bradilalia, bradicardia, intolerancia al frío, constipación, aumento de peso y relajación retardada de reflejos tendinosos; también, se asocia a voz grave, piel y pelo seco o áspero, facies abotagadas y macroglosia, entre otros (tabla 2).

\section{Complicaciones del hipotiroidismo no tratado en la gestación}

El hipotiroidismo puede tener resultados adversos en el embarazo, y van a depender de la gravedad de la alteración (hipotiroidismo manifiesto, hipotiroidismo subclínico o hipotiroxinemia aislada). En el hipotiroidismo manifiesto se ha demostrado una relación clara con preeclampsia e hipertensión arterial [58], desprendimiento de placenta [59], parto prematuro (incluso antes de las 32 semanas), bajo peso al nacer, aumento de la tasa de cesáreas, hemorragia postparto [60], mortalidad perinatal y deterioro cognitivo del neonato [61].

En cuanto al hipotiroidismo subclínico, se ha encontrado una relación no tan clara con la mayoría de los desenla- 


\begin{tabular}{|c|c|c|}
\hline Tejido u órgano & Signo o síntoma & Causa \\
\hline Piel & $\begin{array}{l}\text { - Pálidez, carotenemia o frialdad } \\
\text { - Resequedad } \\
\text { - Vitiligo o alopecia areata } \\
\text { - Edema sin fóvea }\end{array}$ & $\begin{array}{l}\text { - Menor flujo sanguíneo } \\
\text { - Menor secreción acinar } \\
\text { - Autoinmunidad } \\
\text { - Mixedema por acumulación } \\
\text { de proteínas y agua en la piel }\end{array}$ \\
\hline Cabello y uñas & Áspero o quebradizo/as & $\begin{array}{l}\text { Aumento de depósitos de } \\
\text { glicosaminoglicanos }\end{array}$ \\
\hline Hematológico & $\begin{array}{l}\text { - Sangrado } \\
\text { - Anemia normocítica o } \\
\text { macrocitosis }\end{array}$ & $\begin{array}{l}\text { - EVW tipo } 1 \\
\text { - Disminución de la masa eritroide }\end{array}$ \\
\hline Cardíaco & $\begin{array}{l}\text { - Reducción del gasto cardíaco } \\
\text { - Hipercolesterolemia }\end{array}$ & $\begin{array}{l}\text { - Regulación de genes implicados } \\
\text { en la contractilidad } \\
\text { - Menor metabolismo del } \\
\text { colesterol }\end{array}$ \\
\hline Respiratorio & $\begin{array}{l}\text { Hipoventilación o disnea } \\
\text { de esfuerzo }\end{array}$ & Debilidad de músculos respiratorios \\
\hline Intestinal & $\begin{array}{l}\text { - Constipación } \\
\text { - Atrofia gástrica } \\
\text { - NASH }\end{array}$ & Menor movimiento intestinal \\
\hline Muscular & $\begin{array}{l}\text { Atrofia muscular, hiperuricemia } \\
\text { y dolor articular }\end{array}$ & $\begin{array}{l}\text { Menor actividad y disminución } \\
\text { del filtrado renal }\end{array}$ \\
\hline Otros & $\begin{array}{l}\text { - Hiponatremia } \\
\text { - Síndrome del túnel carpiano } \\
\text { e hiperhomocisteinemia }\end{array}$ & $\begin{array}{l}\text { - Depuración de agua alterada } \\
\text { - Aumento de depósitos de } \\
\text { glicosaminoglicanos }\end{array}$ \\
\hline $\begin{array}{l}\text { Coma } \\
\text { mixedematoso }\end{array}$ & $\begin{array}{l}\text { Manifestación severa del } \\
\text { hipotiroidismo }\end{array}$ & Todas las descritas \\
\hline
\end{tabular}

NASH: esteatohepatitis no alcohólica; EVW: enfermedad de von Willebrand.

ces descritos, debido a los diferentes puntos de corte de TSH utilizados y a la disponibilidad de medición de antiTPO [62]. Esto es importante, ya que las mujeres con hipotiroidismo subclínico poseen mayor tendencia a desenlaces adversos cuando hay presencia de antiTPO, incluso a niveles menores de TSH [62]. En las guías de la ATA, las mujeres con $\mathrm{TSH}>2,5 \mathrm{mUI} / \mathrm{L}$ con anti-TPO positivos, presentaron complicaciones relacionadas al embarazo, no así para aquellas con $\mathrm{TSH}>4 \mathrm{mUI} / \mathrm{L}$ con antiTPO negativos [63]. En el hipotiroidismo subclínico se ha encontrado una relación positiva con complicaciones en el embarazo, respecto a las eutiroideas. Existe mayor riesgo de parto prematuro (OR=1,29; IC95\% 1,01-1,64) [64], pérdida del embarazo $(R R=2,01$; IC95\% 1,66-2,44), abruptio de placenta $(R R=2,14 ; I C 95 \% 1,23-3,70)$, y muerte neonatal ( $R R=2,58$; IC95\% 1,41-4,73) [65]. Hay resultados conflictivos con relación al hipotiroidismo subclínico y el deterioro neurocognitivo. Estudios observacionales sugieren una relación, pero la heterogeneidad de los resultados está dada por los niveles de elevación de TSH y las diferentes herramientas usadas para evaluar el desarrollo cognitivo $[66,67]$. 
Respecto a la hipotiroxinemia aislada, los resultados perinatales y neonatales no están claros. En el estudio del consorcio de investigación FASTER (del inglés, First And Second Trimester Evaluation of Risk) se encontró un aumento en el parto prematuro $(\mathrm{OR}=1,62 ; \mathrm{IC} 95 \%$ 1,00-2,62), macrosomía (OR=1,97; IC95\% $1 ; 37-2,83)$ y diabetes gestacional (OR=1,70; IC95\% 1,02-2,84) [68]. También se ha relacionado con el parto prematuro (OR=1,46; IC95\% 1,12-1;90) [64], y resultados conflictivos respecto al desarrollo neurocognitivo $[48,67]$.

\section{Beneficios del tratamiento del hipotiroidismo manifiesto}

La relación entre el hipotiroidismo manifiesto y los desenlaces adversos en el embarazo está claramente establecida, ahora bien, por motivos éticos no se ha desarrollado un ensayo clínico para evaluar los efectos de la reposición hormonal. Con todo esto, está demostrado que las manifestaciones y complicaciones se relacionan con los niveles de la TSH [69], haciendo indispensable el reemplazo de hormona tiroidea en hipotiroidismo manifiesto durante el embarazo.

\section{Beneficios del tratamiento del hipotiroidismo subclínico}

El reemplazo de hormona tiroidea ha mostrado desenlaces mixtos en el subgrupo de la población embarazada con hipotiroidismo subclínico. Se ha demostrado que la suplencia hormonal en mujeres con anti-TPO positivos e hipotiroidismo subclínico logra reducir la tasa de partos prematuros de un $29 \%$ a un $5 \%$, primordialmente en el subgrupo con TSH >4 mUI/L [70]. Estudios como el de Negro y colaboradores, con estrategias de tamización universal en las que se le asigna tratamiento a mu- jeres con hipotiroidismo subclínico, contra aquellas no tratadas, han logrado demostrar menores tasas de aborto, parto pretérmino, abruptio de placenta, y preeclampsia, entre otras [70]. Asimismo, un metaanálisis reciente encontró menor pérdida de embarazo en el grupo tratado, con riesgo de sesgo por la poca presentación de eventos y la heterogeneidad de los estudios ( $\mathrm{OR}=0,51$; IC95\% 0,25-1,05) [71].

Pequeños estudios han hallado también el beneficio de la terapia de reemplazo con beneficio potencial temprano en el embarazo (primer trimestre), en aquellos con hipotiroidismo con $\mathrm{TSH}>2,5 \mathrm{mUI} / \mathrm{L}$ y $<4 \mathrm{mUI} / \mathrm{L}$, con antiTPO positivos, sobre todo en cuanto a la pérdida del embarazo $[37,72]$. Aun así, el reemplazo hormonal no ha demostrado lograr mejoría en los desenlaces cognitivos de este grupo de pacientes $[73,74]$. No está claro si está en relación al reemplazo tardío en la mayoría de los estudios (después de la semana 12), o al tipo y momento de la prueba aplicada. Por lo tanto, el reemplazo hormonal en esta población sigue siendo conflictivo $[5,75]$.

A pesar de las limitaciones en la evidencia disponible, el tratamiento con levotiroxina sugiere un beneficio, de predominio en aquellas con anti-TPO positivos, por lo cual la ATA generó en 2017 una recomendación en este subgrupo de población gestante, dependiente de los niveles de anticuerpos (figura 2).

Respecto a la hipotiroxinemia aislada, no se han encontrado diferencias en los desenlaces al recibir tratamiento en cuanto a parto prematuro, preeclampsia, hipertensión gestacional, tasa de aborto espontáneo o desarrollo neurológico $[74,76]$. Esto posiblemente también esté en relación al inicio tardío en la reposición hormonal en ambos estu- 


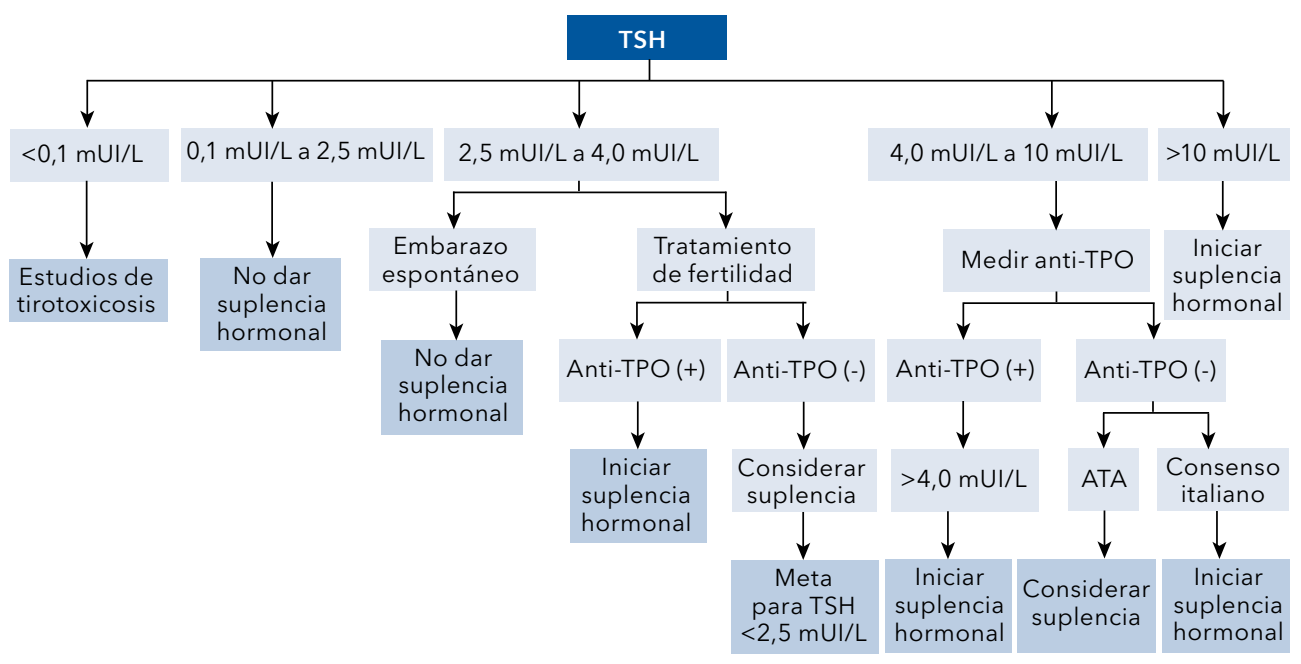

Figura 2. Algoritmo de tratamiento del hipotiroidismo en el embarazo. Anti-TPO: anticuerpos antiperoxidasa tiroidea; TSH: hormona estimulante de la tiroides; ATA: American Thyroid Association.

dios; a pesar de todo, no existe suficiente evidencia para recomendar el tratamiento en la hipotiroxinemia aislada.

\section{Tamización gestacional}

Como se ha descrito, el hipotiroidismo manifiesto y subclínico se asocia a complicaciones gestacionales y perinatales. La realización de medición hormonal es económica y fácilmente realizable, por lo cual existe un intenso interés en determinar el beneficio de la tamización universal gestacional. Sin embargo, el cribado de mujeres asintomáticas es controvertido, porque no se ha logrado demostrar que logre disminuir los desenlaces adversos a nivel global [76]. Con la tamización de la población de alto riesgo de padecer hipotiroidismo (tabla 3), se estima que se pasan por alto un tercio de las mujeres con hipotiroidismo subclínico o manifiesto. Aun así, en la tamización de la población de bajo riesgo, cuando se detecta y administra tratamiento para el hipotiroidismo manifiesto o subclí- nico, se asocia a menos complicaciones $[77,78]$. Datos limitados sugieren que la tamización o cribado universal puede ser más rentable que el basado en riesgos, con una relación de costoefectividad incremental de 7.258 dólares americanos por año de vida ajustado por calidad [77].

En Colombia se recomienda la tamización universal a todas las mujeres gestantes. Sin embargo, no se menciona nada respecto a la medición de anticuerpos [79]. La ATA no recomienda medir de manera rutinaria los niveles de T4L por la gran variabilidad en el embarazo; se prefiere tomar la decisión de tratamiento con los niveles de TSH y anti-TPO, que han sido los estudiados en los desenlaces materno-fetales [2].

Se recomienda la tamización inicial con niveles de TSH; en caso de no contar con rangos poblacionales, se propone un rango normal gestacional de 0,4 mUI/L a 4,0 mUI/L. Si es un embarazo espontáneo no requiere ningún tipo de intervención; en trata- 
Tabla 3. Condiciones de alto riesgo para padecer hipotiroidismo en el embarazo

Síntomas de hipotiroidismo

Antecedentes familiares o personales de enfermedad tiroidea

Presencia de anti-TPO y/o bocio

Edad $>30$ años

Diabetes mellitus tipo 1

Aborto espontáneo recurrente, parto prematuro o esterilidad

Múltiples embarazos previos (>2)

Irradiación de cabeza y cuello

Obesidad con IMC $>40 \mathrm{~kg} / \mathrm{m}^{2}$

Cirugía de tiroides o cuello

Uso de amiodarona o litio

Uso reciente de contrastes yodados

Zona geográfica con déficit moderado o severo de yodo

IMC: índice de masa corporal; anti-TPO: anticuerpos antiperoxidasa tiroidea.

mientos de fertilidad se recomiendan valores $<2,5 \mathrm{mUI} / \mathrm{L}$, y se puede considerar tratamiento en aquellas con TSH $>2,5 \mathrm{mUI} / \mathrm{L}$. Si la TSH es superior a 4,0 $\mathrm{mUI} / \mathrm{L}$, se recomienda realizar la medición de anti-TPO para guiar la decisión terapéutica.

\section{Tratamiento del hipotiroidismo gestacional}

La terapia del hipotiroidismo debe estar dirigida a alcanzar la mitad inferior de las metas propuestas para la población si existen los rangos de referencia, de lo contrario se propone mantener la $\mathrm{TSH}$ menor a $2,5 \mathrm{mUI} / \mathrm{L}$, con rango superior de $4 \mathrm{mUI} / \mathrm{L}$. Cuando el valor inicial se encuentra entre $2,5 \mathrm{mUI} / \mathrm{L}$ y 4 $\mathrm{mUI} / \mathrm{L}$ no se requiere intervención adicional, no obstante, se ha demostrado un beneficio de la suplencia en aquellas pacientes dentro de este rango con anti-TPO positivos, que se encuentran bajo terapia de fertilidad asistida, con metas de TSH $<2 \mathrm{mUI} / \mathrm{L}[2,66]$.

Si el valor de TSH es superior a 10 $\mathrm{mUI} / \mathrm{L}$, se recomienda dar tratamiento independiente de los niveles de anti-TPO. Sin embargo, existe un rango intermedio entre $4 \mathrm{mUI} / \mathrm{L}$ y $10 \mathrm{mUI} / \mathrm{L}$, donde la ATA propone la medición de anti-TPO, que en caso de ser positivos, las pacientes deben recibir suplencia hormonal para prevenir la progresión a hipotiroidismo manifiesto y sus complicaciones, ya discutidas previamente $[2,66]$ (figura 2 ), pero en caso de resultar negativos, se sugiere dejarlo a juicio médico; recomendación que fue cuestionada por el panel experto italiano (IEP, del inglés, Italian Expert Panel), en el que proponen tratamiento a todas las gestantes con TSH en este rango [80]. El IEP considera que se le atribuye mucho valor solo a los anticuerpos, porque por el estado de inmunotolerancia relativo (incluso en el primer trimestre), pueden ser negativos en el embarazo, sobre todo en mujeres con niveles previos de anticuerpos bajos; tal como lo demostró el estudio de Rago y colaboradores, donde una paciente con anti-Tg y anti-TPO circulantes negativos, y ecografía con patrón tiroideo heterogéneo hipoecoico, desarrolló hipotiroidismo subclínico a los 24 meses de seguimiento [81]. Llama la atención que los dos estudios en los que la ATA basó la recomendación, no mostraron beneficio de tratamiento, pero iniciaron tarde (semana 13 y 16) la reposición hormonal, como se expuso previamente $[69,74]$. Posterior al análisis del costo-beneficio, el IEP recomienda el uso de reemplazo hormonal, dado que no hay una evidencia robusta a favor o en contra del reemplazo hormonal en pacientes con hipotiroidismo subclínico con anti-TPO negativos, sopesando el riesgo de sobretratar a un pequeño grupo de pacientes [80]. 
El tratamiento se recomienda con levotiroxina oral, debido a que por el efecto de las desyodinasas, el feto no recibe adecuadamente levotiroxina-T3 o el tratamiento con tiroides desecado [2,82].

\section{Hipotiroidismo pregestacional}

En las mujeres gestantes con hipotiroidismo previo, la glándula tiroides no logra compensar satisfactoriamente las demandas de T4, las cuales aumentan en la primera mitad del embarazo. En estas se requiere un aumento de la dosis de levotiroxina hasta del $85 \%$ para evitar el desarrollo de hipotiroidismo materno [83], iniciándose tan pronto se documente el embarazo (primera menstruación faltante). El incremento va a depender del control pregestacional y de la causa del hipotiroidismo, pero se recomienda que en mujeres que vienen eutiroideas, se incremente en un $30 \%$ la dosis, para simular la fisiología normal en el embarazo [83].

\section{Seguimiento de la función tiroidea}

Se recomienda hacer un seguimiento de la función tiroidea a todas las mujeres con factores de riesgo para desarrollar hipotiroidismo en el embarazo (tabla 3) de manera mensual, hasta por lo menos la semana 30 [2]. Una vez que culmine el embarazo, se debe retornar a la dosis pregestacional (si no tenía suplencia, se suspende), y se realiza control a las 6 semanas posparto para definir el estado final tiroideo.

\section{Conclusiones}

Las alteraciones del eje tiroideo en el embarazo son una entidad relativamente frecuente, que impacta clínicamente tanto en el bienestar materno como en el fetal. Un adecuado conocimiento de la fisiología y los cambios normales del eje hormonal es fundamental para el correcto diagnóstico y seguimiento de la disfunción. Al tener un comportamiento diferente a la población no gestante, las mediciones hormonales pueden llegar a confundir al médico tratante y llevar a un diagnóstico o terapéutica errada.

Finalmente, conviene mencionar que muchas de las complicaciones derivadas del hipotiroidismo gestacional (manifiesto, subclínico o hipotiroxinemia aislada), culminan en patologías que pueden llegar a comprometer la vida materna o fetal, en donde una adecuada y oportuna medición de un examen económico y ampliamente disponible, como lo es la determinación de la TSH, puede cambiar el curso natural de la enfermedad al realizarse intervención farmacológica temprana.

\section{Referencias}

1. Moleti M, Trimarchi F, Vermiglio F. Thyroid physiology in pregnancy. Endocr Pract 2014;20:589596. https://doi.org/10.4158/ep13341.Ra.

2. Alexander EK, Pearce EN, Brent GA, Brown RS, Chen H, Dosiou C, et al. 2017 Guidelines of the American Thyroid Association for the diagnosis and management of thyroid disease during pregnancy and the postpartum. Thyroid 2017;27:315-389. https://doi. org/10.1089/thy.2016.0457.

3. Morais N, Assis ASA, Corcino CM, Saraiva DA, Berbara T, Ventura CDD, et al. Recent recommendations from ATA guidelines to define the upper reference range for serum TSH in the first trimester match reference ranges for pregnant women in Rio de Janeiro. Arch Endocrinol Metab 2018;62:386-391. https://doi. org/10.20945/2359-3997000000064.

4. De Groot $L$, Abalovich M, Alexander EK, Amino N, Barbour L, Cobin RH, et al. Management of thyroid dysfunction during pregnan- 
cy and postpartum: an Endocrine Society clinical practice guideline. J Clin Endocrinol Metab 2012;97:2543-2565. https://doi.org/10.1210/ jc.2011-2803.

5. Ge GM, Leung MTY, Man KKC, Leung WC, Ip P, Li GHY, et al. Maternal thyroid dysfunction during pregnancy and the risk of adverse outcomes in the offspring: A systematic review and metaanalysis. J Clin Endocrinol Metab 2020;105. https://doi.org/10.1210/clinem/dgaa555.

6. Springer D, Jiskra J, Limanova Z, Zima T, Potlukova E. Thyroid in pregnancy: From physiology to screening. Crit Rev Clin Lab Sci 2017;54:102-116. https://doi.org/10.1080/104 08363.2016.1269309.

7. Yazbeck CF, Sullivan SD. Thyroid disorders during pregnancy. Med Clin North Am 2012;96:235-256. https://doi.org/10.1016/j. mcna.2012.01.004.

8. Jarvis S, Nelson-Piercy C. Thyroid disease in pregnancy. In: Gonik B, Weiner C, James D, Steer $\mathrm{P}$, Robson $\mathrm{S}$, eds. High-risk pregnancy: Management options. Cambridge: Cambridge University Press; 2017. p. 1191-1216.

9. Hume R, Simpson J, Delahunty C, van Toor H, Wu SY, Williams FL, et al. Human fetal and cord serum thyroid hormones: developmental trends and interrelationships. J Clin Endocrinol Metab 2004;89:4097-4103. https://doi.org/10.1210/ jc.2004-0573.

10. Glinoer $D$, de Nayer $P$, Bourdoux $P$, Lemone M, Robyn C, van Steirteghem A, et al. Regulation of maternal thyroid during pregnancy. J Clin Endocrinol Metab 1990;71:276-287. https://doi. org/10.1210/jcem-71-2-276.

11. Hershman JM. Physiological and pathological aspects of the effect of human chorionic gonadotropin on the thyroid. Best Pract Res Clin Endocrinol Metab 2004;18:249-265. https://doi. org/10.1016/j.beem.2004.03.010.

12. Ballabio M, Poshychinda M, Ekins RP. Pregnancy-induced changes in thyroid function: role of human chorionic gonadotropin as putative regulator of maternal thyroid. J Clin Endocrinol Metab 1991;73:824-831. https://doi. org/10.1210/jcem-73-4-824.

13. Grün JP, Meuris $S$, De Nayer $P$, Glinoer $D$. The thyrotrophic role of human chorionic gonadotrophin (hCG) in the early stages of twin (versus single) pregnancies. Clin Endocrinol (Oxf) 1997;46:719-725. https://doi.org/10.1046/ j.1365-2265.1997.2011011.x.

14. Maia AL, Goemann IM, Meyer EL, Wajner SM. Deiodinases: the balance of thyroid hormone: type 1 iodothyronine deiodinase in human physiology and disease. J Endocrinol 2011;209:283297. https://doi.org/10.1530/joe-10-0481.

15. Kester MH, Kaptein E, Van Dijk CH, Roest TJ, Tibboel D, Coughtrie MW, et al. Characterization of iodothyronine sulfatase activities in human and rat liver and placenta. Endocrinology 2002;143:814-819. https://doi. org/10.1210/endo.143.3.8686.

16. Silva JF, Ocarino NM, Serakides R. Thyroid hormones and female reproduction. Biol Reprod 2018;99:907-921. https://doi.org/10.1093/biolre/ioy115

17. Dentice $\mathbf{M}$, Marsili A, Zavacki A, Larsen $\mathbf{P R}$, Salvatore D. The deiodinases and the control of intracellular thyroid hormone signaling during cellular differentiation. Biochim Biophys Acta 2013;1830:3937-3945. https://doi. org/10.1016/j.bbagen.2012.05.007.

18. Ferreiro B, Bernal J, Goodyer CG, Branchard

CL. Estimation of nuclear thyroid hormone receptor saturation in human fetal brain and lung during early gestation. J Clin Endocrinol Metab 1988;67:853-856. https://doi.org/10.1210/ jcem-67-4-853.

19. López-Muñoz E, Mateos-Sánchez L, MejíaTerrazas GE, Bedwell-Cordero SE. Hypothyroidism and isolated hypothyroxinemia in pregnancy, from physiology to the clinic. Taiwan J Obstet Gynecol 2019;58:757-763. https://doi. org/10.1016/j.tjog.2019.09.005.

20. Loubière LS, Vasilopoulou E, Bulmer JN, Taylor PM, Stieger B, Verrey F, et al. Expression of thyroid hormone transporters in the human placenta and changes associated with intrauterine growth restriction. Placenta 2010;31:295-304. https://doi.org/10.1016/j.placenta.2010.01.013.

21. Fantz CR, Dagogo-Jack S, Ladenson JH, Gronowski AM. Thyroid function during pregnancy. Clin Chem 1999;45:2250-2258.

22. Zhang $X$, Li $C$, Mao J, Wang $W$, Xie $X$, Peng $\mathbf{S}$, et al. Gestation-specific changes in maternal thyroglobulin during pregnancy and lactation in an iodine-sufficient region in China: a longitu- 
dinal study. Clin Endocrinol (Oxf) 2017;86:229235. https://doi.org/10.1111/cen.13175.

23. Sirakov M, Skah S, Nadjar J, Plateroti M. Thyroid hormone's action on progenitor/stem cell biology: new challenge for a classic hormone? Biochim Biophys Acta 2013;1830:3917-3927. https://doi.org/10.1016/j.bbagen.2012.07.014.

24. Burrow GN, Fisher DA, Larsen PR. Maternal and fetal thyroid function. N Engl J Med 1994;331:1072-1078. https://doi.org/10.1056/ nejm199410203311608.

25. Institute of Medicine. Dietary reference intakes: Applications in dietary assessment. Washington, DC: The National Academies Press; 2000. ISBN: 978-0-309-07183-3.

26. Wang Z, Li C, Teng Y, Guan Y, Zhang L, Jia X, et al. The effect of iodine-containing vitamin supplementation during pregnancy on thyroid function in late pregnancy and postpartum depression in an iodine-sufficient area. Biol Trace Elem Res 2020;198:1-7. https://doi.org/10.1007/ s12011-020-02032-y.

27. World Health Organization (WHO), International Council for the Control of the lodine Deficiency Disorders, United Nations Children's Fund. Assessment of iodine deficiency disorders and monitoring their elimination: a guide for programme managers. 3rd ed. Geneva: World Health Organization; 2007.

28. Völzke H, Erlund I, Hubalewska-Dydejczyk A, Ittermann T, Peeters RP, Rayman M, et al. How do we improve the impact of iodine deficiency disorders prevention in europe and beyond? Eur Thyroid J 2018;7:193-200. https://doi. org/10.1159/000490347.

29. Vargas-Uricoechea H, Murillo-Palacios J, Ramírez-Bejarano LE. Análisis del estado poblacional del yodo en Colombia y la necesidad de modificar los niveles de yodación universal de la sal. Revista Colomb Endocrinología Diabetes Metab 2020;7:87-98. https://doi.org/10.53853/ encr.7.2.608.

30. Kwak-Kim J, Bao S, Lee SK, Kim JW, GilmanSachs A. Immunological modes of pregnancy loss: inflammation, immune effectors, and stress. Am J Reprod Immunol 2014;72:129-140. https:// doi.org/10.1111/aji.12234.

31. Morelli S MM, Goldsmith LT, Kashani BN, Ponzio NM. The maternal immune system during pregnancy and its influence on fetal development. Res Rep Biol 2015;6:171-189. https://doi. org/https://doi.org/10.2147/RRB.S80652.

32. Kim NY, Cho HJ, Kim HY, Yang KM, Ahn HK, Thornton S, et al. Thyroid autoimmunity and its association with cellular and humoral immunity in women with reproductive failures. Am J Reprod Immunol 2011;65:78-87. https://doi. org/10.1111/j.1600-0897.2010.00911.x.

33. Weetman AP. Diseases associated with thyroid autoimmunity: explanations for the expanding spectrum. Clin Endocrinol (Oxf) 2011;74:411-418. https://doi.org/10.1111/ j.1365-2265.2010.03855.x.

34. Shields BM, Knight BA, Hill AV, Hattersley AT, Vaidya B. Five-year follow-up for women with subclinical hypothyroidism in pregnancy. J Clin Endocrinol Metab 2013;98:E1941-1945 https://doi.org/10.1210/jc.2013-2768.

35. Nambiar V, Jagtap VS, Sarathi V, Lila AR, Kamalanathan S, Bandgar TR, et al. Prevalence and impact of thyroid disorders on maternal outcome in Asian-Indian pregnant women. J Thyroid Res 2011;2011:429097. https://doi. org/10.4061/2011/429097.

36. De Leo S, Pearce EN. Autoimmune thyroid disease during pregnancy. Lancet Diabetes Endocrinol 2018;6:575-586. https://doi.org/10.1016/ s2213-8587(17)30402-3.

37. Negro R, Formoso G, Mangieri T, Pezzarossa A, Dazzi D, Hassan H. Levothyroxine treatment in euthyroid pregnant women with autoimmune thyroid disease: effects on obstetrical complications. J Clin Endocrinol Metab 2006;91:2587-2591. https://doi.org/10.1210/ jc.2005-1603.

38. Mammen JS, Cappola AR. Autoimmune thyroid disease in women. JAMA 2021;325:2392-2393. https://doi.org/10.1001/jama.2020.22196.

39. Bagis T, Gokcel A, Saygili ES. Autoimmune thyroid disease in pregnancy and the postpartum period: relationship to spontaneous abortion. Thyroid 2001;11:1049-1053. https://doi. org/10.1089/105072501753271743.

40. Pradhan M, Anand B, Singh N, Mehrotra M. Thyroid peroxidase antibody in hypothyroidism: it's effect on pregnancy. J Matern Fetal Neonatal Med 2013;26:581-583. https://doi.or $\mathrm{g} / 10.3109 / 14767058.2012 .745498$ 
41. Toulis KA, Goulis DG, Venetis CA, Kolibianakis EM, Negro R, Tarlatzis BC, et al. Risk of spontaneous miscarriage in euthyroid women with thyroid autoimmunity undergoing IVF: a meta-analysis. Eur J Endocrinol 2010;162:643652. https://doi.org/10.1530/eje-09-0850.

42. Thangaratinam S, Tan A, Knox E, Kilby MD, Franklyn J, Coomarasamy A. Association between thyroid autoantibodies and miscarriage and preterm birth: meta-analysis of evidence. Bmj 2011;342:d2616. https://doi.org/10.1136/ bmj.d2616.

43. He $X$, Wang $P$, Wang $Z$, He $X, X u$ D, Wang B. Thyroid antibodies and risk of preterm delivery: a meta-analysis of prospective cohort studies. Eur J Endocrinol 2012;167:455-464. https://doi. org/10.1530/eje-12-0379.

44. Negro R. Thyroid autoimmunity and pre-term delivery: brief review and meta-analysis. J Endocrinol Invest 2011;34:155-158. https://doi. org/10.1007/bf03347047.

45. Kumru $P$, Erdogdu E, Arisoy R, Demirci $O$, Ozkoral A, Ardic C, et al. Effect of thyroid dysfunction and autoimmunity on pregnancy outcomes in low risk population. Arch Gynecol Obstet 2015;291:1047-1054. https://doi.org/10.1007/ s00404-014-3533-9.

46. Wasserman EE, Nelson $K$, Rose NR, Eaton W, Pillion JP, Seaberg E, et al. Maternal thyroid autoantibodies during the third trimester and hearing deficits in children: an epidemiologic assessment. Am J Epidemiol 2008;167:701-710. https://doi.org/10.1093/aje/kwm342.

47. Williams FLR, Watson J, Ogston SA, Visser TJ, Hume R, Willatts P. Maternal and umbilical cord levels of T4, FT4, TSH, TPOAb, and TgAb in term infants and neurodevelopmental outcome at 5.5 years. J Clin Endocrinol Metab 2013;98:829838. https://doi.org/10.1210/jc.2012-3572.

48. Ghassabian A, Bongers-Schokking JJ, de Rijke YB, van Mil N, Jaddoe VW, de Muinck Keizer-Schrama SM, et al. Maternal thyroid autoimmunity during pregnancy and the risk of attention deficit/hyperactivity problems in children: the Generation R Study. Thyroid 2012;22:178-186. https://doi.org/10.1089/ thy.2011.0318.

49. Bestwick JP, John R, Maina A, Guaraldo V, Joomun M, Wald NJ, et al. Thyroid stimulating hormone and free thyroxine in pregnancy: expressing concentrations as multiples of the median (MoMs). Clin Chim Acta 2014;430:33-37. https://doi.org/10.1016/j.cca.2013.12.030.

50. La'ulu SL, Roberts WL. Ethnic differences in first-trimester thyroid reference intervals. Clin Chem 2011;57:913-915. https://doi. org/10.1373/clinchem.2010.161240.

51. Männistö T, Surcel HM, Ruokonen A, Vääräsmäki M, Pouta A, Bloigu A, et al. Early pregnancy reference intervals of thyroid hormone concentrations in a thyroid antibody-negative pregnant population. Thyroid 2011;21:291298. https://doi.org/10.1089/thy.2010.0337.

52. Lee RH, Spencer CA, Mestman JH, Miller EA, Petrovic I, Braverman LE, et al. Free T4 immunoassays are flawed during pregnancy. Am J Obstet Gynecol 2009;200:260-266. https://doi. org/10.1016/j.ajog.2008.10.042.

53. Soldin OP, Tractenberg RE, Hollowell JG, Jonklaas J, Janicic N, Soldin SJ. Trimesterspecific changes in maternal thyroid hormone, thyrotropin, and thyroglobulin concentrations during gestation: trends and associations across trimesters in iodine sufficiency. Thyroid 2004;14:1084-1090. https://doi.org/10.1089/ thy.2004.14.1084.

54. Yue B, Rockwood AL, Sandrock T, La'ulu SL, Kushnir MM, Meikle AW. Free thyroid hormones in serum by direct equilibrium dialysis and online solid-phase extraction--liquid chromatography/tandem mass spectrometry. Clin Chem 2008;54:642-651. https://doi. org/10.1373/clinchem.2007.098293.

55. Allan WC, Haddow JE, Palomaki GE, Williams JR, Mitchell ML, Hermos RJ, et al. Maternal thyroid deficiency and pregnancy complications: implications for population screening. J Med Screen 2000;7:127-130. https://doi.org/10.1136/jms.7.3.127.

56. Blatt AJ, Nakamoto JM, Kaufman HW. National status of testing for hypothyroidism during pregnancy and postpartum. J Clin Endocrinol Metab 2012;97:777-784. https://doi. org/10.1210/jc.2011-2038.

57. Smith TJ, Bahn RS, Gorman CA. Connective tissue, glycosaminoglycans, and diseases of the thyroid. Endocr Rev 1989;10:366-391. https:// doi.org/10.1210/edrv-10-3-366. 
58. Leung AS, Millar LK, Koonings PP, Montoro M, Mestman JH. Perinatal outcome in hypothyroid pregnancies. Obstet Gynecol 1993;81:349-353.

59. Männistö T, Mendola P, Grewal J, Xie Y, Chen Z, Laughon SK. Thyroid diseases and adverse pregnancy outcomes in a contemporary US cohort. J Clin Endocrinol Metab 2013;98:27252733. https://doi.org/10.1210/jc.2012-4233.

60. Idris I, Srinivasan R, Simm A, Page RC. Maternal hypothyroidism in early and late gestation: effects on neonatal and obstetric outcome. Clin Endocrinol (Oxf) 2005;63:560-565. https://doi. org/10.1111/j.1365-2265.2005.02382.x.

61. Willoughby KA, McAndrews MP, Rovet JF. Effects of maternal hypothyroidism on offspring hippocampus and memory. Thyroid 2014;24:576-584. https://doi.org/10.1089/ thy.2013.0215.

62. Liu H, Shan Z, Li C, Mao J, Xie X, Wang W, et al. Maternal subclinical hypothyroidism, thyroid autoimmunity, and the risk of miscarriage: a prospective cohort study. Thyroid 2014;24:1642 1649. https://doi.org/10.1089/thy.2014.0029.

63. Korevaar TI, Schalekamp-Timmermans S, de Rijke YB, Visser WE, Visser W, de Muinck Keizer-Schrama SM, et al. Hypothyroxinemia and TPO-antibody positivity are risk factors for premature delivery: the generation R study. J Clin Endocrinol Metab 2013;98:4382-4390. https:// doi.org/10.1210/jc.2013-2855.

64. Korevaar TIM, Derakhshan A, Taylor PN, Meima M, Chen L, Bliddal S, et al. Association of thyroid function test abnormalities and thyroid autoimmunity with preterm birth: A systematic review and meta-analysis. JAMA 2019;322:632 641. https://doi.org/10.1001/jama.2019.10931.

65. Maraka S, Singh-Ospina NM, O'Keeffe DT, Rodriguez-Gutierrez R, Espinosa-De Ycaza $\mathbf{A E}, \mathbf{W i} \mathbf{C l}$, et al. Effects of increasing levothyroxine on pregnancy outcomes in women with uncontrolled hypothyroidism. Clin Endocrinol (Oxf) 2017;86:150-155. https://doi. org/10.1111/cen.13168.

66. Rotondi M, Capelli V, Chiovato L, Nappi RE. 2017 ATA guidelines on the management of thyroid dysfunctions in pregnancy: what do OB/GYNs need to know? Gynecol Endocrinol 2019;35:276-279. https://doi.org/10.1080/095 13590.2018 .1532496
67. Nelson SM, Haig C, McConnachie A, Sattar N, Ring SM, Smith GD, et al. Maternal thyroid function and child educational attainment: prospective cohort study. BMJ 2018;360:k452. https://doi.org/10.1136/bmj.k452.

68. Cleary-Goldman J, Malone FD, LambertMesserlian G, Sullivan L, Canick J, Porter TF, et al. Maternal thyroid hypofunction and pregnancy outcome. Obstet Gynecol 2008;112:85-92. https://doi.org/10.1097/ AOG.0b013e3181788dd7.

69. Taylor PN, Minassian C, Rehman A, Iqbal A, Draman MS, Hamilton W, et al. TSH levels and risk of miscarriage in women on long-term levothyroxine: a community-based study. J Clin Endocrinol Metab 2014;99:3895-3902. https:// doi.org/10.1210/jc.2014-1954.

70. Nazarpour S, Ramezani Tehrani F, Amiri M, Bidhendi Yarandi R, Azizi F. Levothyroxine treatment and pregnancy outcomes in women with subclinical hypothyroidism: a systematic review and meta-analysis. Arch Gynecol Obstet 2019;300:805-819. https://doi. org/10.1007/s00404-019-05245-2.

71. Bein M, Yu OHY, Grandi SM, Frati FYE, Kandil I, Filion KB. Levothyroxine and the risk of adverse pregnancy outcomes in women with subclinical hypothyroidism: a systematic review and meta-analysis. BMC Endocr Disord 2021;21:34. https://doi.org/10.1186/s12902021-00699-5.

72. Negro R, Schwartz A, Gismondi R, Tinelli A, Mangieri T, Stagnaro-Green A. Increased pregnancy loss rate in thyroid antibody negative women with TSH levels between 2.5 and 5.0 in the first trimester of pregnancy. J Clin Endocrinol Metab 2010;95:e44-48. https://doi. org/10.1210/jc.2010-0340

73. Zhou Q, Wang $\mathbf{C}, \mathbf{X u} \mathbf{H}, \mathbf{L i} \mathbf{X}$. Impact of preconception treatment initiation for hypothyroidism on neurocognitive function in children. J Clin Endocrinol Metab 2020;105: e3919-3928. https://doi.org/10.1210/clinem/dgaa565.

74. Dhillon-Smith RK, Middleton LJ, Sunner KK, Cheed V, Baker K, Farrell-Carver S, et al. Levothyroxine in women with thyroid peroxidase antibodies before conception. N Engl J Med 2019;380:1316-1325. https://doi.org/10.1056/ NEJMoa1812537. 
75. Casey BM, Thom EA, Peaceman AM, Varner MW, Sorokin Y, Hirtz DG, et al. Treatment of subclinical hypothyroidism or hypothyroxinemia in pregnancy. N Engl J Med 2017;376:815-825. https://doi.org/10.1056/ NEJMoa1606205.

76. Bath SC, Rayman MP. Antenatal thyroid screening and childhood cognitive function. N Engl J Med 2012;366:1640-1641. https://doi. org/10.1056/NEJMc1202720.

77. Negro R, Schwartz A, Gismondi R, Tinelli A, Mangieri T, Stagnaro-Green A. Universal screening versus case finding for detection and treatment of thyroid hormonal dysfunction during pregnancy. J Clin Endocrinol Metab 2010;95:1699-1707. https://doi.org/10.1210/ jc.2009-2009.

78. Jouyandeh Z, Hasani-Ranjbar S, Qorbani M, Larijani B. Universal screening versus selective case-based screening for thyroid disorders in pregnancy. Endocrine 2015;48:116-123. https://doi.org/10.1007/s12020-014-0385-9.

79. Gómez-Sanchez P, Arévalo-Rodríguez I, Collazos-Vidal C. Guías de práctica clínica para la prevención, detección temprana y tratamiento de las complicaciones del embarazo, parto o puerperio. Centro Nacional de Investigación en Evidencia y Tecnologías en Salud (CINETS), Ministerio de Salud y Protección Social. Guías No. 11-15. Bogotá D.C.: Ministerio de Salud y Protección Social-Colciencias; 2013. Acceso 15 de agosto de 2021. Disponible en https://www. minsalud.gov.co/sites/rid/Lists/BibliotecaDigital/RIDE/INEC/IETS/Gu\%C3\%ADa.completa. Embarazo.Parto.2013.pdf.

80. Rotondi M, Chiovato L, Pacini F, Bartalena L, Vitti P. Management of subclinical hypothyroidism in pregnancy: A comment from the Italian Society of Endocrinology and the Italian Thyroid Association to the 2017 American Thyroid Association guidelines-"the Italian way". Thyroid 2018;28:551-555. https://doi. org/10.1089/thy.2017.0424.

81. Rago T, Chiovato L, Grasso L, Pinchera A, Vitti P. Thyroid ultrasonography as a tool for detecting thyroid autoimmune diseases and predicting thyroid dsfunction in apparently healthy subjects. J Endocrinol Invest 2001;24:763-769. https://doi.org/10.1007/bf03343925.

82. Biondi B, Bartalena L, Cooper DS, Hegedüs L, Laurberg P, Kahaly GJ. The 2015 European Thyroid Association Guidelines on diagnosis and treatment of endogenous subclinical hyperthyroidism. Eur Thyroid J 2015;4:149-163. https://doi.org/10.1159/000438750.

83. Alexander EK, Marqusee E, Lawrence J, Jarolim P, Fischer GA, Larsen PR. Timing and magnitude of increases in levothyroxine requirements during pregnancy in women with hypothyroidism. N Engl J Med 2004;351:241249. https://doi.org/10.1056/NEJMoa040079. 